\title{
O uso de smart speakers na incapacidade: Uma scoping review
}

\author{
Rafael Tavares', Helena Sousa ${ }^{2}$ e Jaime Ribeiro, 3 \\ 1Escola Superior de Saúde, Politécnico de Leiria, Leiria, Portugal | rgst93@gmail.com | \\ https://orcid.org/0000-0002-1128-290X \\ ${ }^{2}$ Centro de Estudos do Movimento e Atividade Humana, (CEMAH), Departamento de \\ Terapia Ocupacional, Politécnico do Porto, Porto, Portugal | hes@ess.ipp.pt| \\ https://orcid.org/0000-0002-0461-1883 \\ ${ }^{3}$ Centro de Investigação em Didática e Tecnologia na Formação de Formadores (CIDTFF), \\ Universidade de Aveiro, Aveiro, Portugal; Center for Innovative Care and Health, Escola \\ Superior de Saúde, Technology (ciTechCare), Politécnico de Leiria, Leiria, Portugal | \\ jaime.ribeiro@ipleiria.pt | https://orcid.org/0000-0002-1548-5579
}

\begin{abstract}
Introdução: Os smart speakers (SS), como o Amazon Echo e o Google Nest, estão estabelecidos no mercado mundial e disponíveis para a população geral. A inteligência artificial programada nestes dispositivos de conversação e a sua compatibilidade com diferentes redes cria oportunidades para a criação de intervenções em diferentes populações com incapacidade aplicáveis num contexto de casa inteligente. Objetivos: Compreender como os SS estão a ser utilizados por indivíduos com incapacidade, revelar o seu potencial e possibilidades de intervenção. Métodos: Em fevereiro de 2021 foi realizada uma scoping review baseada em artigos das bases de dados PubMed/Medline, B-On, Scopus e Web of Science. Foram considerados artigos publicados desde 2016 até 2021, escritos em português e inglês e revistos por pares. Foram incluídos estudos focados na utilização de SS disponíveis no mercado geral (Google Nest / Home, Amazon Echo, Invoke, HomePod) como agente de intervenção no ambiente residencial ou ECU, em populações com condições diversas, abrangendo indivíduos com deficiências sensoriais, cognitivas, emocionais e motoras. Resultados: Foram identificadas diferentes abordagens e exigências dos utilizadores. Os dispositivos foram aplicados como uma solução singular ou como um elemento de sistemas de maior complexidade, interagindo com outros dispositivos ou softwares. Conclusões: A identificação de diferentes intervenções para diferentes incapacidades sublinha o potencial de intervenção destes dispositivos com melhorias funcionais observadas em incapacidades físicas, cognitivas, sensoriais e emocionais. Estes dispositivos podem substituir dispositivos dispendiosos criados para nichos de população e sistemas fechados, dirigidos para formas concretas de incapacidade. Sendo a personalização uma característica relevante, a aplicação de SS não dispensa o acompanhamento técnico no processo.
\end{abstract}

Palavras-chave: Deficiência; Internet of Things; Casa Inteligente; Sistemas Ativados por Voz.

\section{The Use of Smart Speakers in Disability: A Scoping Review}

Abstract: Introduction: Smart speakers, like Amazon Echo and Google Nest, are established in the worldwide mainstream market, being available to the general population. The artificial intelligence programmed within these conversational devices and their compatibility with different networks creates opportunities for a wide range of interventions in different populations with disability, in a smart home context; Goals: To understand how smart speakers are being used by individuals with disability, uncovering possibilities of intervention and considering their potential; Methods: A scoping review of articles present in PubMed/Medline, B-On, Scopus and Web of Science databases in February 2021 was conducted. Articles published since 2016 until 2021, written in Portuguese and English and peer reviewed were considered. Studies exploring the use of general market available smart speakers (Google Nest / Home, Amazon Echo, Invoke, HomePod) as an home intervention agent or environmental control unit, focusing on individuals with sensorial, cognitive, emotional and motor impairment were included; Results: Different approaches and user demands were identified. Devices were applied as a single solution or as an element of bigger systems, interacting with other devices or software's; Conclusions: The identification of different interventions for different disabilities underlines the intervention potential of these devices with observed functional improvements in physical, cognitive, sensory, and emotional disabilities. These improvements can promote the replacement of expensive devices created for niche populations and closed systems developed with specific goals in a specific form of disability. As customization is a relevant feature, the application of SS does not dispense with technical support in the process.

Keywords: Disability; Internet of Things; Smart Home; Voice Activated Systems. 


\section{Introdução}

Nos últimos sete anos, foram lançados no mercado mundial smart speakers (SS), que beneficiam a população em geral. Estes altifalantes inteligentes incluem voice assistants (VA) que recorrem a inteligência artificial (Al), como a Siri, da Apple, agora utilizada no dispositivo HomePod, e a Cortana da Microsoft, compatível com o Harman Kardon Invoke (Hoy, 2018; Kepuska \& Bohouta, 2018). Outros foram desenvolvidos simultaneamente com os SS, a exemplo do Google Assistant, a inteligência artificial programada no Google Home e mais recentemente no Google Nest, bem como a Amazon Alexa, associada aos aparelhos Amazon Echo (Kafle et al., 2021; Kepuska \& Bohouta, 2018). Os SS estão em expansão no mercado convencional e várias marcas desenvolveram os seus próprios dispositivos, com o Mi Smart Speaker da Xiaomi e o anunciado Galaxy Home da Samsung a serem destacados pelas suas compatibilidades com dispositivos próprios associados, inseridos no conceito Internet of Things (IoT) (Ashfaq et al., 2021; Ling et al., 2021; Wortmann \& Flüchter, 2015).

De facto, os VA surgiram no mercado antes dos SS, o que significa que embora os SS dependam dos VA para funcionar, o oposto não é necessariamente verdade. Em 2010, a Apple introduziu os VA no mercado em geral lançando a Siri como uma aplicação autónoma, incorporada nos sistemas operativos iOS no ano seguinte (Guzman, 2017). Em 2014 a Google lançou o Google Now e a Microsoft criou a Cortana, enquanto a Amazon se preparava para lançar o primeiro SS, o Amazon Echo, que incorpora a assistente Alexa (Hoy, 2018; Riccardi, 2014). A importante marca subsequente consistiu no lançamento do Google Home em 2016, com a substituição simultânea do Google Now pelo Google Assistant (Li et al., 2017). SS e VA estão indubitavelmente associados, mas são muitas vezes confundidos.

\subsection{Voice Assistants}

Os VA consistem em software conversacional com inteligência artificial. A Al é tradicionalmente definida como a exibição de inteligência por máquinas e, apesar das diferenças de perspetiva entre programadores, a sua evolução converge no sentido do pensamento racional assemelhado ao dos seres humanos, bem como ação racional em linha com a ação humana. Um agente inteligente só está completo com a ligação entre a $\mathrm{Al}$, plataformas sensíveis a variações, e ainda elementos de efeito. Os agentes inteligentes estão, desta forma, capacitados para a deteção de variações ambientais e a ação conforme (Russell et al., 1995).

Qualquer software precisa de um hardware, uma plataforma onde possa funcionar e manifestar-se. Para os VA, essas plataformas eram inicialmente computadores ou smartphones. Ainda que estes dispositivos continuem a ser uma opção para a interação com VA hoje em dia, a introdução de SS criou outra alternativa capaz de detetar variações no ambiente - a deteção de palavras-chave, no mínimo - desenvolver um raciocínio a partir das interações seguintes e efetuar ações como consequência, reunindo os três princípios de um agente inteligente.

Alguns dos principais usos dos VA na população geral neste momento consistem na realização de pesquisas, stream de música, interações baseadas na loT, consulta de agenda, e definição de temporizadores, alertas e despertadores (Ammari et al. 2019).

A literatura sugere que os SS promovem um maior envolvimento com os VA, quando comparadas com outros equipamentos, e que as ações requeridas vão são compatíveis com as características destes interfaces (Bentley et al., 2018). Por estas razões, para além do facto de a utilização de SS libertar o smartphone para uma utilização simultânea sem alterações no visor, o que permite ao utilizador manter a tarefa sem variações visuais, a presente revisão considerará apenas estes dispositivos.

A personificação do diálogo é reconhecida como um fator atrativo para promover o envolvimento e a satisfação com os dispositivos (Bentley et al., 2018; Pradhan et al., 2018). 
Este reconhecimento tem provocado um aumento do interesse de utilizadores com incapacidade e profissionais de saúde em aplicarem SS em diferentes quadros.

\subsection{Acessibilidade}

A acessibilidade dos VA para pessoas com incapacidade e determinação a fiabilidade da interação corresponde a uma área emergente de interesse. Ballati et al., 2018; sugerem que a Siri, Alexa e Google Assistant apresentam um rácio de reconhecimento semelhante para indivíduos com disartria, com os três a variar entre $50 \%$ a $60 \%$. Contudo, diferem em alguns aspetos considerados relevantes. Os autores identificaram que Siri tenta sempre dar uma resposta, mesmo que em total disparidade com o tema pretendido, enquanto a Alexa e Google Assistant apenas respondem a frases compreendidas. Outro aspeto relevante é que a Alexa frequentemente não responde a comandos de uma só palavra, o que pode constituir uma barreira para as pessoas com dificuldades de expressão oral.

Masina et al., 2020 avaliaram a funcionalidade física, linguística e cognitiva dos indivíduos em estudo e propuseram o Mini-Mental State Examination (MMSE) e Robertson Dysarthria Profile como preditores do desempenho do utilizador na interação com o dispositivo Google Home. Neste trabalho é sugerido que indivíduos com incapacidade podem interagir com o dispositivo, desde que sejam atingidos requisitos mínimos de competências cognitivas e linguísticas medidas nos instrumentos mencionados.

\subsection{Controlo Ambiental}

Diferentes Environment Control Units (ECU) têm sido desenvolvidas e utilizadas com populações com incapacidades desde os anos 60 até aos dias de hoje (Noda, 2018). A maioria destes sistemas receberam atualizações para melhorar as suas respostas, ou substituídos por modelos mais recentes e com uma melhor resposta. No entanto, algumas barreiras persistiram ao longo do tempo, tais como a necessidade de assistência técnica, limitações gramaticais e de vocabulário, necessidades de formação para uma aplicação efetiva do sistema, dificuldade em alargar a sua ação a outros fins que não aqueles para que foram desenvolvidos, baixa capacidade de integração de elementos externos complementares, baixa disseminação no mercado e/ou altos valores de mercado e consequentes dificuldades de financiamento (Judge et al., 2009; Noda, 2018).

Os SS consistem numa oportunidade atual de desenvolver novas intervenções para pessoas com incapacidade, transportar a terapia para o ambiente doméstico e assim aumentar o tempo de contacto terapêutico (caso a utilização do dispositivo seja mediada por um técnico), reinventar ECU com preços mais baixos e sistemas abertos, e até proporcionar um acompanhamento omnipresente, se garantida a necessária privacidade de dados gerados (Masina et al., 2020; Noda, 2018).

\subsection{Presente Estudo}

O facto de estes dispositivos oferecerem uma grande quantidade de possibilidades a explorar em contexto doméstico, com diferentes intervenções, diferentes populações e diferentes resultados, levou à necessidade de desenvolver um estudo com um alcance amplo e contrastante com uma revisão sistemática, proporcionando dados aprofundados relativos a uma única forma de intervenção para um resultado específico.

Foi realizada uma pesquisa preliminar nas bases de dados Cochrane e PubMed e não foram identificadas quaisquer scoping reviews, revisões sistemáticas ou integrativas. Pelo que se considera a necessidade de desenvolvimento do presente estudo para uma investigação estruturada do estado da ciência neste campo.

Esta revisão tem o objetivo de compreender como os SS estão a ser utilizados por indivíduos com incapacidade, revelar possibilidades de intervenção e considerar o seu potencial. 
Vol. 8 | Investigação Qualitativa em Saúde: Avanços e Desafios

Foi realizada alicerçada na metodologia Joanna Briggs Institute (JBI), que nos permitiu mapear as provas disponíveis neste campo. Almeja identificar os tipos de provas disponíveis, lacunas de conhecimento, e clarificar conceitos e definições chave na literatura (Aromatis\& Munn, 2020).

\section{Questão de Revisão}

A presente revisão alicerça-se na questão: "Como é que os smart speakers disponíveis no mercado são utilizados por indivíduos com incapacidade para promover a função num ambiente doméstico inteligente?". Esta questão foi formada respeitando uma estrutura PCC (População, Conceito, Contexto), para alargar o âmbito da investigação, quando comparada com a estrutura mais circunscrita da PICO (População, Intervenção, Comparador, Outcome). Dada a possibilidade de interação entre SS e dispositivos inteligentes desenvolvidos considerando o conceito de loT, foi formada uma sub-questão PCC: "Que tarefas e atividades podem os smart speakers facilitar ao serem utilizados como unidades de controlo ambiental por indivíduos com limitações motoras?".

\section{Palavras-chave}

Deficiência; Internet of Things; Casa Inteligente; Sistemas Ativados por Voz.

\section{Critérios de elegibilidade}

\subsection{Participantes}

Este estudo explora a utilização de SS por pessoas com incapacidade/ deficiência, sendo elegíveis estudos centrados em indivíduos com deficiência sensorial, cognitiva, emocional e motora, desde que garantida a capacidade de falar e interagir com o dispositivo. Considerando a evidência que mostra que os SS presentes no mercado e a sua IA correspondente, não detetam a totalidade das solicitações de indivíduos com limitações de expressão oral, foram excluídos os estudos com populações com esta e outras perturbações da fala. Do mesmo modo, não foram considerados estudos cujas populações possam necessitar de uso regular de ventiladores ou que se encontrem dependentes de terceiros para a sua remoção, ainda que num uso mais reduzido.

\subsection{Conceito}

Foram incluídos artigos que exploram a utilização de SS disponíveis no mercado geral (Google Nest/Home, Amazon Echo, Invoke, HomePod) como um agente de intervenção doméstica ou ECU em populações com incapacidade. Foram excluídos estudos focados na segurança de dados, aspetos técnicos de engenharia da tecnologia e o potencial destes dispositivos na gestão da energia doméstica, sem qualquer menção à aplicação junto de pessoas com incapacidade.

\subsection{Contexto}

Foram incluídos estudos desenvolvidos em qualquer ponto do globo, que consideram a aplicação em ambiente doméstico/residencial, ou laboratorial tendo em vista a generalização aos anteriormente mencionados. 


\subsection{Fontes}

Esta scoping review considerou desenhos de estudos experimentais e quaseexperimentais. Foram ainda considerados para inclusão estudos observacionais analíticos e descritivos, incluindo estudos de caso.

\section{Métodos}

\subsection{Estratégia de Pesquisa}

Foram pesquisadas as seguintes bases de dados PubMed/Medline, B-On, Scopus e Web of Science. A pesquisa e a extração de dados decorreram em Fevereiro de 2021.

Tendo em consideração que os SS foram disponibilizados no mercado convencional com - lançamento do primeiro dispositivo Amazon Echo em 2014, e que a sua utilização aumentou após a entrada do dispositivo Google Home no mercado em 2016, foram incluídos nesta revisão estudos publicados desde 2016 até 2021, escritos em português e inglês e revistos por pares. Foram utilizados os seguintes termos de pesquisa associados a elementos de truncagem "smart*home", "smart*speaker" e "disabilit*".

\subsection{Seleção de Evidência}

Os metadados dos motores de busca foram reunidos no software Mendeley Desktop 1.19.4, para serem atribuídos a pastas correspondentes a cada base de dados. Foram gerados ficheiros Research Information Systems (ris.), posteriormente importados no software WebQDA 3.0. Neste, os duplicados foram removidos e os restantes títulos analisados segundo os critérios de elegibilidade acima descritos e atribuídos a pastas de inclusão/ exclusão. Artigos dúbios foram discutidos e ponderados por pelo menos dois investigadores.

Os resultados da execução dos passos metodológicos descritos encontram-se resumidos em diagrama referente ao processo de seleção e motivos de exclusão (Figura 1). Após aplicação dos critérios, foram considerados elegíveis cinco artigos: (Boumpa et al., 2019; Ghazal et al., 2019; Hugo et al., 2021; Smith et al., 2020; Wallace \& Morris, 2018).

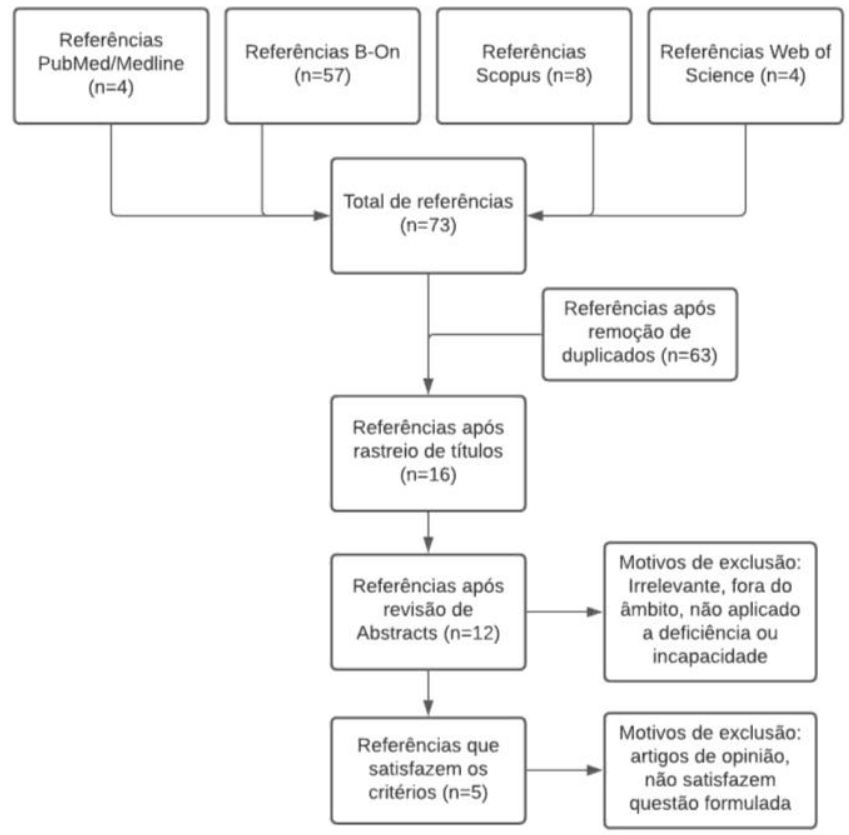

Fig. 1. Processo de seleção. 


\section{Análise de dados e Apresentação}

A evidência incluída foi produzida na América do Norte $(n=2)$, Médio Oriente $(n=1)$ e Europa $(n=2)$. Apesar da abertura para incluir artigos publicados desde 2016, apenas se verificaram elegíveis artigos publicados a partir de 2018, sendo um desse ano, dois publicados em 2019, um em 2020 e um no ano corrente, 2021. Considerando as filiações dos autores, os artigos provieram de áreas de medicina física e reabilitação $(n=1)$, engenharia $(n=2)$, informática $(n=1)$ e psicologia $(n=1)$.

Tabela 1. Descrição de estudos selecionados.

\begin{tabular}{|c|c|c|c|c|c|c|}
\hline Autores & $\begin{array}{c}\text { Ano de } \\
\text { publicaç } \\
\text { ão }\end{array}$ & Título & $\begin{array}{c}\text { Objetivo } \\
\text { do } \\
\text { estudo }\end{array}$ & $\begin{array}{l}\text { Participa } \\
\text { ntes }\end{array}$ & $\begin{array}{c}\text { Intervençã } \\
\mathbf{0}\end{array}$ & SS \\
\hline $\begin{array}{c}\text { Boumpa, } \\
\text { E.; } \\
\text { Gkogkidis } \\
\text {, A.; } \\
\text { Charalam } \\
\text { pou, I.; } \\
\text { Ntaliani, } \\
\text { A.; } \\
\text { Kakaroun } \\
\text { tas, A.; } \\
\text { Kokkinos, } \\
\text { V. }\end{array}$ & 2019 & $\begin{array}{c}\text { An } \\
\text { Acoustic- } \\
\text { Based } \\
\text { Smart } \\
\text { Home } \\
\text { System } \\
\text { for } \\
\text { People } \\
\text { Suffering } \\
\text { from } \\
\text { Dementia }\end{array}$ & $\begin{array}{l}\text { Reconhe } \\
\text { cimento } \\
\text { de } \\
\text { pessoas } \\
\text { através } \\
\text { de um } \\
\text { sistema } \\
\text { de ajuda } \\
\text { à } \\
\text { memória } \\
\text { baseado } \\
\text { no som. }\end{array}$ & $\begin{array}{l}\text { Indivíduo } \\
\text { s com } \\
\text { diagnósti } \\
\text { co de } \\
\text { demência }\end{array}$ & $\begin{array}{l}\text { Desenvolvi } \\
\text { mento de } \\
\text { um sistema } \\
\text { ubíquo de } \\
\text { mapeament } \\
\text { o de } \\
\text { dispositivos } \\
\text { conectados } \\
\text { à mesma } \\
\text { rede WiFi. } \\
\text { Quando um } \\
\text { dispositivo } \\
\text { familiar é } \\
\text { detetado, o } \\
\text { SS produz } \\
\text { um som } \\
\text { pré- } \\
\text { programado } \\
\text { exclusivame } \\
\text { nte } \\
\text { associado } \\
\text { ao } \\
\text { proprietário } \\
\text { do } \\
\text { dispositivo } \\
\text { Desenvolvi } \\
\text { mento de } \\
\text { um sistema } \\
\text { com recurso } \\
\text { a SS, uma } \\
\text { câmara de } \\
\text { vigilância e } \\
\text { sensores. } \\
\text { Uma vez } \\
\text { ativado, o } \\
\text { sistema lê e } \\
\text { processa a } \\
\text { informação } \\
\text { do } \\
\text { seguida de } \\
\text { descricão } \\
\text { aubiente, }\end{array}$ & $\begin{array}{c}\text { Não } \\
\text { identifi } \\
\text { cado }\end{array}$ \\
\hline
\end{tabular}


Vol. 8 | Investigação Qualitativa em Saúde: Avanços e Desafios

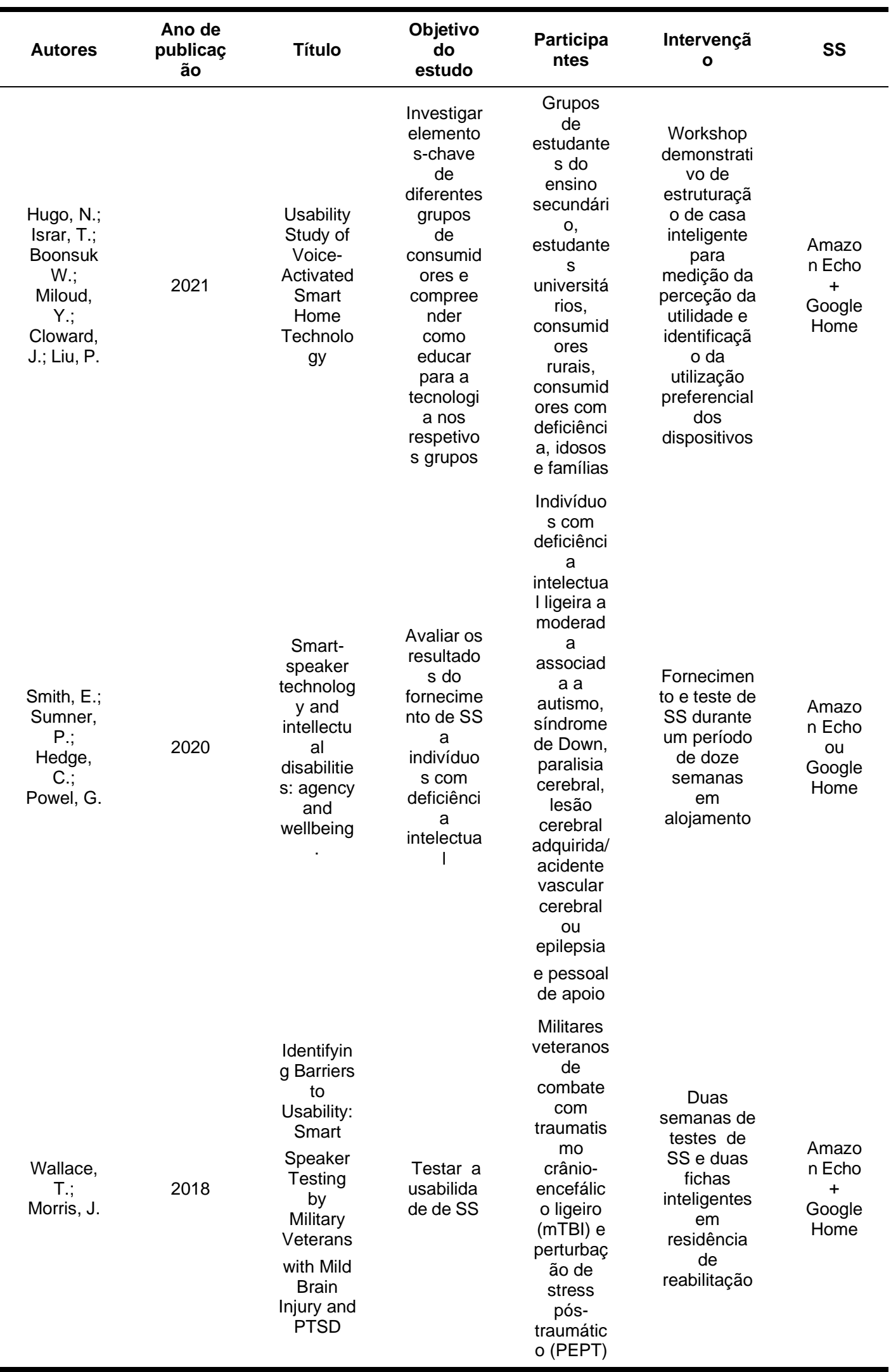




\subsection{Smart Speakers Selecionados e Características Correspondentes}

Os SS da Amazon e Google foram as escolhas mais populares nesta revisão. Nenhum estudo incluiu especificamente outros modelos como HomePod, Invoke, Galaxy Home e Mi Smart Speaker. Apesar deste facto, dois estudos (Boumpa et al., 2019; Ghazal et al., 2019) utilizaram o SS como elemento de um sistema desenvolvido e, em ambos os casos, os autores não discriminaram um modelo concreto utilizado, circunstância que abre a possibilidade de que os dispositivos mencionados possam funcionar nos sistemas criados, desde que assegurada a capacidade de interagir com o smartphone do utilizador e aplicações ou sistemas de mapeamento desenvolvidas pelos investigadores.

Smith et al., (2020) forneceram o Amazon Echo ou Google Home a cada um dos seus participantes com deficiência intelectual submetidos a intervenção. Após esta atribuição, estudaram as diferenças entre os novos utilizadores, um grupo de controlo e um terceiro grupo constituído por participantes que já utilizavam SS no início da intervenção.

Os restantes estudos identificados na Tabela 1. (Hugo et al., 2021; Wallace \& Morris, 2018) exploraram os dispositivos Amazon Echo e Google Home de forma alternada, promovendo uma comparação. Wallace \& Morris, (2018) avaliaram a preferência de utilizadores com traumatismo crânio-encefálico ligeiro (mTCE) e perturbação de stress pós-traumático (PSPT) após a intervenção. A maioria dos sujeitos reportou uma preferência pelo dispositivo Amazon Echo, considerando que era mais fácil de configurar inicialmente, embora possivelmente associado à qualidade da ligação WiFi das residências em que os dispositivos foram testados, e que o Google Home necessitava de mais assistência. O controlo manual de volume foi mencionado como uma dificuldade associada ao dispositivo Google Home, uma vez que requer motricidade fina, que pode ser comprometida em quadros de comprometimento físico e/ ou sensorial. Apesar da preferência geral pelo dispositivo da Amazon, os autores relatam que o Google Assistant foi mais eficaz na compreensão dos comandos de voz. Características como som, voz do assistente, palavra de ativação e estética foram também relatadas como mais bem classificadas no dispositivo Echo.

Hugo et al., (2021) compararam características antes da intervenção em diferentes grupos populacionais, incluindo um grupo com incapacidades não especificadas, iniciando essa comparação pelos assistentes. Os autores identificaram vantagens nas características da Alexa, nomeadamente a possibilidade de personalização, realização de compras por voz, e ainda a possibilidade de realizar chamadas ou enviar uma mensagem escrita convertida a partir de uma mensagem oral, algo que o Google Assistant também oferece, mas através de um smartphone associado. Este estudo também diferencia modelos, através da comparação entre diferentes versões, em cada marca. Apesar de se verificar que o Echo Show é a versão globalmente mais completa entre os dispositivos Echo, este não apresenta o hub ZigBee encontrado no Echo Plus. Este hub é uma característica a considerar se a intenção é estabelecer ligações com outros dispositivos ZigBee e assim desenvolver uma casa inteligente. Não é, no entanto, imperativo para este fim, uma vez que um hub externo pode ser adquirido e ligado à mesma rede WiFi, o que viabiliza a ligação de dispositivos ZigBee mesmo com o Echo Dot, a versão com menos características catalogadas. Os dispositivos Google são igualmente compatíveis com estes hubs externos. Os autores compararam as mais recentes colunas inteligentes Google disponíveis no mercado, o Google Nest Hub e o Google Home Mini, hoje conhecido como Google Nest Mini. É de referir que nestas versões se verifica uma simplificação da forma de controlo manual de volume, representando uma melhoria da limitação identificada por Wallace \& Morris, (2018). O dispositivo Nest Hub apresenta um ecrã táctil que promove a interação direta e aumenta a informação visual, para além de adicionar um Chromecast incorporado, tornando possível interagir com uma televisão e completar ações como aumentar o volume, mudar de canal e iniciar um serviço de streaming. Tal como o hub ZigBee, o Chromecast também pode ser adquirido como um dispositivo externo, compatível com diferentes speakers. 


\subsection{Interação}

A literatura revista sugere que os SS podem funcionar sozinhos ou ser simplesmente uma parte, um interface interativo de um sistema aberto e mais complexo. Tais sistemas são construídos de acordo com as necessidades dos utilizadores e podem ser compostos exclusivamente por dispositivos disponíveis no mercado, como os acima mencionados, ou podem incluir elementos autodesenvolvidos.

A compatibilidade com aplicações smartphone e wi-fi, ZigBee e outras redes, aumenta as possibilidades de controlo do ambiente. O contacto com uma aplicação de controlo remoto para televisão, como complemento ou alternativa aos dispositivos de controlo televisivo, é um exemplo sugerido (Hugo et al., 2021). A eletricidade doméstica pode ser manipulada através de lâmpadas, interruptores, tomadas e extensões inteligentes com ligação wi-fi (Hugo et al., 2021; Wallace \& Morris, 2018). O utilizador pode assim controlar não só a iluminação ambiental, mas também a ativação e desativação de dispositivos. A interação de termóstatos inteligentes foi sugerida para poupar custos, uma vez que estes podem ler medições térmicas e mediar o funcionamento de dispositivos de regulação térmica, como aquecedor, ventoinha ou ar condicionado, em conformidade com os dados recolhidos (Hugo et al., 2021). Apesar de se demonstrar um potencial interessante para esta utilização final, tal automatização é também um recurso que vale a pena explorar na deficiência, uma vez que pode promover economia de esforço, reduzindo as exigências cognitivas e a necessidade de se exercerem esforços físicos para manutenção das condições ambientais. Foram também mencionados vários dispositivos relacionados com a segurança, nomeadamente câmaras, detetores de movimento, sensores de contacto, que representam um conjunto de dispositivos versáteis que podem ser aplicados noutras utilizações que não a segurança, como automatizações adicionais.

Ghazal et al., (2019) aproveitam a versatilidade das câmaras de segurança e utilizam-nas para fazer leituras visuais do ambiente. Estas leituras são posteriormente analisadas num servidor desenvolvido, a fim de fornecer uma descrição audível, a indivíduos com deficiência visual, através do SS. O SS pode igualmente ser utilizado para pedir essa informação, desencadeando uma nova leitura e processamento de dados para descrição. O sistema doméstico acústico apresentado por Boumpa et al., (2019) integra um SS enquanto elemento de um sistema de maior complexidade. Neste estudo, foi desenvolvido um Software as a Service (SaaS) para atuar como um servidor onde se regista informação para a identificação de doentes com demência, os seus familiares, dispositivos de cada indivíduo, um som pessoal associado e os SS presentes na habitação. Quando um dispositivo registado de um familiar se liga à rede habitacional do indivíduo, o router comunica com o servidor, que por sua vez associa o dispositivo ao familiar que o utiliza. Neste ponto recupera a informação selecionada, incluindo o som exclusivo daquele familiar, localizando ainda o SS mais próximo do indivíduo nessa altura. O SS reproduz então o som, de modo a estimular a memória acústica do indivíduo e assim promover o reconhecimento do familiar que visita.

\subsection{Proatividade Interacional Exigida}

A forma como os dispositivos foram utilizados diversificou, sendo identificadas exigências de interação ativa, passiva e mista. Como esperado, a maioria dos artigos (Hugo et al., 2021; Smith et al., 2020; Wallace \& Morris, 2018) sugeriu uma utilização ativa para o dispositivo, em que o utilizador inicia proativamente a interação dizendo a palavra-chave de ativação. Após a ativação, o utilizador formula uma pergunta ou verbaliza uma instrução, desencadeando uma ação no dispositivo, ou outros dispositivos ligados na rede. Em alternativa, o estudo de Boumpa et al., (2019) apresenta uma utilização passiva. Neste estudo, os autores trabalharam com pessoas que sofrem de demência e programaram o sistema de modo a ter uma natureza e funcionamento ubíquos. Este funcionamento ubíquo garantiu a realização da ação para a qual foi projetado, mesmo que os sujeitos estivessem esquecidos ou momentaneamente inconscientes da presença dos SS. 
Tanto as alternativas ativas como passivas são sugeridas simultaneamente no estudo de Ghazal et al., (2019), no qual um utilizador com deficiência visual tinha como opção de configuração do sistema, um automatismo de descrição sonora do ambiente uma vez detetada a sua presença, ou o acionamento provocado por uma solicitação ao SS, de modo a obter atualizações relativamente a alterações no ambiente ou simplesmente ter acesso a uma repetição da descrição.

\subsection{Ação - Incapacidade}

As aplicações em populações com deficiências cognitivas foram as mais amplamente exploradas. Boumpa et al., (2019) sugerem que os sistemas com estes dispositivos podem ter um papel na estimulação do reconhecimento mnésico de familiares registados no sistema desenvolvido. Tarefas como definir temporizadores, integrar marcações no calendário e organizar listas de compras, foram também consideradas relevantes em estudos revistos. Tanto Smith et al., (2020) como Wallace \& Morris, (2018) sugerem que estas tarefas são úteis para indivíduos com défices de memorização. A não inclusão destas ou outras estratégias compensatórias e formas de apoio pode levar ao esquecimento de realização de determinada atividade ou a uma maior dificuldade de recrutamento de tarefas e pormenores específicos, o que conduz à frustração. Os autores identificam esta frustração como um fator de risco para a ansiedade e/ou depressão, revelando um possível papel na regulação emocional. As análises qualitativas de Smith et al., (2020) reportam outras atividades com influência mais direta no estado emocional, tais como o stream de música, contar anedotas, conversar, jogar jogos simples ou quizzes. Os participantes relataram que uma maior interação levou a um sentimento de acompanhamento, apesar de essa companhia não substituir uma relação humana.

O sistema proposto por Ghazal et al., (2019) mostra que os SS podem ser uma ferramenta relevante para compensar a deficiência sensorial, neste caso visual, com recurso a uma descrição acústica do espaço e objetos nele presentes. No entanto, e considerando a oferta limitada ou nula de produtos disponíveis no mercado convencional desenvolvidos com este ideal compensatório, a utilização de SS para reconhecimento ambiental pode depender de apoio especializado para a programação e desenvolvimento de sistemas adaptados.

A interação com outros dispositivos inteligentes - que não exclusivamente o smartphone conectados a uma ou mais redes comuns para o controlo do ambiente, foi identificada em dois estudos, embora não tenha sido profundamente explorada pelos autores. As características dos SS sugerem um potencial para agir como unidades de controlo ambiental em casas inteligentes, aumentando a acessibilidade para utilizadores com deficiências motoras. $O$ controlo da iluminação foi explorado através de candeeiros ligados a fichas inteligentes no trabalho de Wallace \& Morris, (2018), e a iluminação inteligente no estudo de Hugo et al., (2021), que propõe outros dispositivos, mas para poupança de energia.

\subsection{Abordagens}

Esta revisão levou à identificação de diferentes abordagens para a inclusão de SS em ambiente doméstico. A maioria dos estudos explora estes dispositivos para a compensação do défice, facilitando atividades com exigências físicas, cognitivas, sensoriais, e até emocionais. No entanto, foi também identificada a aplicação para criação ou aumento de oportunidades de participação em atividades de lazer e outras interativas, neste caso, com maior foco em populações com deficiências cognitivas e/ou emocionais para fins de regulação comportamental. 


\section{Conclusões}

A metodologia qualitativa utilizada no presente estudo permitiu identificar aplicabilidades dos SS em diferentes tipos de incapacidade e em primeira pessoa, proporcionando dados relativos à pertinência das intervenções existentes e potenciais pontos exploratórios para a implementação destes dispositivos enquanto ferramenta de intervenção. Os dispositivos foram utilizados para compensação de deficiências visuais, cognitivas e físicas, sendo que a sua natureza de agente conversacional é também explorada para prestar um acompanhamento em situações de cariz emocional, assim como as potencialidades oferecidas relacionadas com lazer.

Um número crescente de aparelhos eletrodomésticos inteligentes está a emergir no mercado e as pessoas com incapacidade têm agora a oportunidade de criar ecossistemas inteligentes com automatismos e controlo total sobre dispositivos inteligentes através da voz. Os SS podem redirecionar uma ordem para que os dispositivos aspirem, façam café, aqueçam ou arrefeçam o ambiente, desumidifiquem um espaço e aqueçam água, para dar alguns exemplos acrescentados às atividades identificadas nesta revisão. Apesar do potencial interativo com dispositivos externos, não foi identificada, nos artigos selecionados, uma variedade elevada de interações propostas.

Os SS foram propostos enquanto ECU essencialmente para controlo de iluminação e dispositivos ligados a tomadas inteligentes, para além de vigilância. Deste modo identificase a necessidade de explorar de forma mais concreta e aprofundada o impacto dos SS e dispositivos loT na incapacidade, nomeadamente em deficiências motoras limitativas. A produção de evidência qualitativa pode facultar dados não estruturados relativos à identificação de atividades realizadas com SS em diferentes âmbitos, bem como à estruturação de sistemas que os incluam e a perceção que os utilizadores têm dos mesmos.

O aumento de evidência para SS enquanto ECU é, na realidade, uma necessidade sintomática e transversal às restantes aplicabilidades identificadas. Dado que os utilizadores se envolvem mais na interação com VA através de SS em comparação com outros dispositivos onde os assistentes podem ser programados, é pertinente a continuidade do estudo com o uso concreto do SS e não apenas com recurso a VA.

\section{Financiamento}

Este trabalho é financiado por Fundos Nacionais através da FCT - Fundação para a Ciência e a Tecnologia, I.P. no âmbito do projeto UIDB/00194/2020.

\section{Referências}

Ammari, T., Kaye, J., Tsai, J. Y., \& Bentley, F. (2019). Music, Search, and loT: How People (Really) Use Voice Assistants. ACM Transactions on Computer-Human Interaction (TOCHI), 26(3), 128. https://doi.org/10.1145/3311956

Aromatis, E., \& Munn, Z. (2020). JBI Manual for Evidence Synthesis. https://doi.org/https://doi.org/10.46658/JBIMES-20-01

Ashfaq, M., Yun, J., \& Yu, S. (2021). My Smart Speaker is Cool! Perceived Coolness, Perceived Values, and Users' Attitude toward Smart Speakers. International Journal of HumanComputer Interaction, 37(6), 560-573. https://doi.org/10.1080/10447318.2020.1841404

Ballati, F., Corno, F., \& Russis, L. De. (2018). “Hey Siri , do you understand me ?”: Virtual Assistants and Dysarthria.

Bentley, F., Luvogt, C., White, B., \& Lottridge, D. (2018). Understanding the Long-Term Use of Smart Speaker Assistants. 2(3).

Boumpa, E., Gkogkidis, A., Charalampou, I., Ntaliani, A., Kakarountas, A., \& Kokkinos, V. (2019). An Acoustic-Based Smart Home System for People Suffering from Dementia. Technologies (2227-7080), 7(1), 29. https://doi.org/10.3390/technologies7010029

Ghazal, M., Basmaji, T., Qasymeh, M., Salim, R., \& Khalil, A. (2019). Localized assistive scene 
understanding using deep learning and the loT. Proceedings - 2019 International Conference on Future Internet of Things and Cloud Workshops, FiCloudW 2019, 53-58. https://doi.org/10.1109/FiCloudW.2019.00023

Guzman, A. L. (2017). Citation: Guzman, A.L. (2017). Making Al safe for humans: A conversation. 69-85.

Hoy, M. B. (2018). Alexa, Siri, Cortana, and More: An Introduction to Voice Assistants. Medical Reference Services Quarterly, 37(1), 81-88. https://doi.org/10.1080/02763869.2018.1404391

Hugo, N., Israr, T., Boonsuk, W., Ben Miloud, Y., Cloward, J., \& Liu, P. P. (2021). Usability Study of Voice-Activated Smart Home Technology. In Advances in Intelligent Systems and Computing. https://doi.org/10.1007/978-3-030-52575-0_54

Judge, S., Robertson, Z., \& Hawley, M. S. (2009). Users' perceptions of environmental control systems. Assistive Technology Research Series, 25, 426-431. https://doi.org/10.3233/978-160750-042-1-426

Kafle, K., Moran, K., Manandhar, S., Nadkarni, A., \& Poshyvanyk, D. (2021). Security in centralized data store-based home automation platforms: A systematic analysis of nest and hue. ACM Transactions on Cyber-Physical Systems, 5(1). https://doi.org/10.1145/3418286

Kepuska, V., \& Bohouta, G. (2018). Next-generation of virtual personal assistants (Microsoft Cortana, Apple Siri, Amazon Alexa and Google Home). 2018 IEEE 8th Annual Computing and Communication Workshop and Conference, CCWC 2018, 2018-Janua(c), 99-103. https://doi.org/10.1109/CCWC.2018.8301638

Li, B., Sainath, T. N., Narayanan, A., Caroselli, J., Bacchiani, M., Misra, A., ... Shannon, M. (2017). Acoustic modeling for Google home. Proceedings of the Annual Conference of the International Speech Communication Association, INTERSPEECH, 2017-August(August), 399-403. https://doi.org/10.21437/Interspeech.2017-234

Ling, H. C., Chen, H. R., Ho, K. K. W., \& Hsiao, K. L. (2021). Exploring the factors affecting customers' intention to purchase a smart speaker. Journal of Retailing and Consumer Services, 59(October 2020), 102331. https://doi.org/10.1016/j.jretconser.2020.102331

Masina, F., Orso, V., Pluchino, P., Dainese, G., Volpato, S., Nelini, C., ... Gamberini, L. (2020). Investigating the Accessibility of Voice Assistants With Impaired Users: Mixed Methods Study. Journal of Medical Internet Research, 22(9), e18431. https://doi.org/10.2196/18431

Noda, K. (2018). Google Home: smart speaker as environmental control unit. Disability and Rehabilitation. Assistive Technology, 13(7), 674-675. https://doi.org/10.1080/17483107.2017.1369589

Pradhan, A., Mehta, K., \& Findlater, L. (2018). “Accessibility Came by Accident” Use of VoiceControlled Intelligent Personal Assistants by People with Disabilities. Proceedings of the 2018 CHI Conference on Human Factors in Computing Systems, 1-13.

Riccardi, G. (2014). Towards healthcare personal agents. RFMIR 2014 - Proceedings of the 2014 ACM Roadmapping the Future of Multimodal Interaction Research Including Business Opportunities and Challenges, Co-Located with ICMI 2014, 53-56. https://doi.org/10.1145/2666253.2666266

Russell, S. J., Norvig, P., Canny, J. F., Malik, J. M., Edwards, D. D., Jonathan, S. J. S., \& Norvig, P. (1995). A Modern Approach. New Jersey: Prentice-Hall, Inc.

Smith, E., Sumner, P., Hedge, C., \& Powell, G. (2020). Smart-speaker technology and intellectual disabilities: agency and wellbeing. Disability and Rehabilitation: Assistive Technology. https://doi.org/10.1080/17483107.2020.1864670

Wallace, T., \& Morris, J. (2018). Identifying barriers to usability: Smart speaker testing by military veterans with mild brain injury and PTSD. In Breaking Down Barriers: Usability, Accessibility and Inclusive Design. https://doi.org/10.1007/978-3-319-75028-6 10

Wortmann, F., \& Flüchter, K. (2015). Internet of Things: Technology and Value Added. Business and Information Systems Engineering, 57(3), 221-224. https://doi.org/10.1007/s12599-0150383-3 